\title{
Accurate iterative analytic solution of the Kapchinskij-Vladimirskij equations for the case of a matched beam
}

\author{
Oscar A. Anderson \\ Lawrence Berkeley National Laboratory, University of California, Berkeley, California 94720, USA
}

(Received 12 June 2006; published 14 March 2007)

\begin{abstract}
The well-known Kapchinskij-Vladimirskij (KV) equations are difficult to solve in general, but the problem is simplified for the matched-beam case with sufficient symmetry. We show that the interdependence of the two KV equations is eliminated, so that only one needs to be solved-a great simplification. We present an iterative method of solution which can potentially yield any desired level of accuracy. The lowest level, the well-known smooth approximation, yields simple, explicit results with good accuracy for weak or moderate focusing fields. The next level improves the accuracy for high fields; we previously showed [Part. Accel. 52, 133 (1996)] how to maintain a simple explicit format for the results. That paper used expansion in a small parameter to obtain results of second-level accuracy. The present paper, using straightforward iteration, obtains equations of first, second, and third levels of accuracy. For a periodic lattice with beam matched to lattice, we use the lattice and beam parameters as input and solve for phase advances and envelope functions. We find excellent agreement with numerical solutions over a wide range of beam emittances and intensities.
\end{abstract}

DOI: 10.1103/PhysRevSTAB.10.034202

PACS numbers: $52.59 . \mathrm{Sa}$

\section{INTRODUCTION}

This paper analyzes matched beams in alternatinggradient (AG) focusing systems. Such beams have smaller excursions than mismatched beams, thus requiring smaller transport apertures, and are the starting point for analysis of mismatched beams. Designers and experimenters who work with AG systems need simple, accurate ways to predict the performance of matched beams. One usually begins with the coupled Kapchinskij-Vladimirskij (KV) equations [1]. For weak focusing fields, solution by the smooth approximation [2-4] is reasonably accurate. Methods of solution for strong fields [5-7] have tended to be indirect or complex. Lee, using a double expansion in focus strength and emittance, demonstrated high accuracy for a special case [8]. Our previous paper [9] presented general results in a simple explicit format, but with only moderate accuracy.

The present paper, summarized in Ref. [10], uses a convenient iteration method to obtain explicit results. High accuracy is demonstrated for a wide range of parameters, which are: the AG field strength; beam emittance; and beam charge or current. These would be small parameters if normalized (cf. Appendix J), but we use physical units throughout. We assume the lattice to be periodic with the beam matched to the lattice, i.e., having the same periodicity. The lattice is assumed symmetric. (Asymmetric cases are treated with a novel numerical technique by Lund et al. in Ref. [11].)

We solve explicitly for average radius [Eq. (34)], peak radius [Eq. (40)], and the phase advances [Eqs. (44) and (47)]. We give these results for three levels of accuracy and complexity. All these formulas apply to arbitrary symmet- ric lattices. They require at most the $3 \mathrm{rd}$ and 5 th harmonics of the lattice profile, becoming simpler for smooth profiles (Appendix G). Envelope functions are given in Appendix E and illustrated in Fig. 2.

\section{A. Summary}

We begin with the coupled KV equations and show in Sec. III that the matching assumption decouples them so that only one equation must be solved. [Although the required symmetry Eq. (5) has been noted before [8,11], we are not aware that it has been used in obtaining a solution.] Section IV expands the $a(z)$ envelope about its mean and then splits the resulting equation into its average part $A$ and periodic part $\rho$. The differential equation for $\rho(z)$ is solved (Sec. V) by iteration. These results are combined to obtain a matching equation for the average radius $A$. This equation is written to various orders of accuracy; our special definition of "order" is given at the end of Sec. V. The results (Sec. VI) are compared to the results of numerical solution of the full KV equations. The first-order case is usually called the smooth approximation. Second- and third-order terms increasingly improve the accuracy. Over a wide range of parameters, our third-order versions provide a great improvement over those previously published [2-6].

Section VII combines results from Secs. V and VI to give the maximum and minimum radii. The phase advances $\sigma$ and $\sigma_{0}$ are given in Sec. VIII. There is a useful approximation for $\sigma_{0}$ in Sec. IX. Appendices F, G, and $\mathrm{H}$ discuss the use of Fourier analysis of the AG focusing function to facilitate the solution. Some exact formulas for the FODO case [Fig. 5] are given in Appendix I. 


\section{THE KV EQUATIONS AND SYMMETRIC LATTICE MODEL}

The KV equations for envelopes $a(z)$ and $b(z)$ are [1]

$$
\begin{aligned}
& a(z)^{\prime \prime}=-K(z) a+\frac{\in^{2}}{a^{3}}+\frac{2 Q}{a+b} \\
& b(z)^{\prime \prime}=+K(z) b+\frac{\in^{2}}{b^{3}}+\frac{2 Q}{a+b},
\end{aligned}
$$

with dimensionless perveance $Q$, emittance $\in$, and lattice focus-strength function $K(z)$. The latter is periodic over the lattice cell length, defined as $2 L$, so that $K(z+2 L)=$ $K(z)$. In practice, the lattice cell has one or two symmetries. The double symmetry occurs if the focus drift spaces are equal in length. In this paper we assume that the function $K(z)$ has odd symmetry at the center of the drift spaces and even symmetry at mid-electrode points. These symmetry points necessarily alternate at intervals $L / 2$. It is convenient in what follows to choose one of the even points for the $z$ origin. (The odd choice is appropriate for the case of unequal drift lengths; that case is not discussed in the present paper.) Then the double symmetry is expressed as

$$
\begin{aligned}
K(L-z) & =K(L+z), \\
K(L / 2-z) & =-K(L / 2+z) .
\end{aligned}
$$

It follows that $K(z)$ is antisymmetric about $3 L / 2$ and that $K(z)$ obeys the shift relations

$$
\begin{aligned}
& K(z+L)=-K(z), \\
& K(z+2 L)=+K(z) .
\end{aligned}
$$

It also follows that

$$
\begin{gathered}
K(-z)=K(z), \\
\langle K\rangle=0 .
\end{gathered}
$$

Equation (3d) confirms the lattice periodicity which was assumed above. Equations (3a) and (3b) indicate that $K(z)$ can be expanded as a cosine series containing only oddnumbered harmonics.

In this paper we solve Eqs. (1) and (2) for the beam envelopes in the special case where the beam is matched, i.e., $a(z)$ and $b(z)$ have the same periodicity as the lattice. The initial conditions with the symmetry of Eq. (3e) are $a^{\prime}(0)=0, b^{\prime}(0)=0, a(0)=a_{0}, b(0)=b_{0}$.

For given $Q$ and $\in$ and given lattice parameters, the beam will only be matched for specific values of $a_{0}$ and $b_{0}$, which we denote by $a_{\mathrm{m}}$ and $b_{\mathrm{m}}$. Then the initial conditions for a matched beam are

$$
a^{\prime}(0)=0, \quad b^{\prime}(0)=0, \quad a(0)=a_{\mathrm{m}}, \quad b(0)=b_{\mathrm{m}},
$$

with $a(2 L)=a_{\mathrm{m}}$ and $b(2 L)=b_{\mathrm{m}}$.

If we substitute $a_{L}(z) \equiv a(z+L)$ and $b_{L}(z) \equiv b(z+L)$ in Eqs. (1) and (2) and use Eq. (3c), we find that $a_{L}(z)$ and $b_{L}(z)$ also satisfy the KV equations with transposed initial conditions: $a_{L}(0)=b_{\mathrm{m}}, b_{L}(0)=a_{\mathrm{m}}$. We deduce that for a matched beam

$$
b(z)=a(z+L),
$$

so that the denominator of the last term in Eq. (1) can be written $a(z)+b(z)=a(z)+a(z+L)$. Then $b(z)$ does not appear and Eq. (1) is decoupled. In Sec. III and Appendix A we find an expression for the function $[a(z)+$ $a(z+L)]^{-1}$ in terms of integrals involving the given lattice function $K(z)$. After that, Eq. (2) is not needed for our matched-beam case. The main work of this paper will be to find $a_{\mathrm{m}}$ and $b_{\mathrm{m}}$, the maximum and minimum values of the envelope - see Secs. IV, V, VI, and VII. First we introduce some definitions.

\section{A. Operators, functions, parameters, derived quantities}

To aid the solution of Eqs. (1) and (2), we define in Table I the operators $\langle\cdots\rangle,\{\cdots\}, \int, \iint$; the functions $h(z)$, $g(z), \delta(z), \rho(z)$; and the constants $k, \alpha, \beta, q, A, K^{\text {eff }}, \Phi$, and $\rho_{\mathrm{m}}$. In Eq. (21), $h_{1}$ is the first Fourier coefficient of $h(z)-$ cf. Appendix F.

TABLE I. Collection of definitions to be used in this paper.

$\begin{aligned}\langle f\rangle & \equiv(1 / 2 L) \int_{0}^{2 L} f(z) d z \\ \{f\} & \equiv f-\langle f\rangle \\ \int \psi & \equiv \int_{0}{ }^{z} \psi\left(z^{\prime}\right) d z^{\prime} \\ \iint \psi & \equiv\left\{\int_{0}{ }^{z} d z^{\prime} \int_{0}^{z^{\prime}} \psi\left(z^{\prime \prime \prime}\right) d z^{\prime \prime}\right\} \\ k & \equiv K(0) \\ h(z) & \equiv K(z) / k \\ g & \equiv \iint h \\ \delta(z) & \equiv \iint\{h g\} \\ A & \equiv\langle a(z)\rangle \\ \rho(z) & \equiv[a(z)-A] / A \\ \alpha & \equiv 3 \in \in^{2} / A^{4} \\ \beta & \equiv \alpha L^{2} / \pi^{2} \\ q & \equiv Q / A^{2} \\ K^{\mathrm{eff}} & \equiv k^{2}\left\langle\left[\int h\right]^{2}\right\rangle \\ \Phi & \equiv 3 k^{2}\left\langle g^{2}\right\rangle \\ \rho_{\mathrm{m}} & \equiv h_{1} k L^{2} / \pi^{2}\end{aligned}$


In Table I, the operator $\langle\cdots\rangle$ performs an average over a cell length $2 L$. The operator $\{\cdots\}$ removes the average part of a periodic function: e.g., $2\left\{\cos ^{2} x\right\}=\{1+\cos 2 x\}=$ $\cos 2 x$. In this paper, $\iint$ operates exclusively on functions obeying Eqs. (3), producing functions with the same symmetries. It integrates twice and removes the average part, if any, of the result. This removal can be implemented by constructing a suitable lower limit for the outer integral. To illustrate, Eq. (12) can be written

$$
g \equiv \int_{L / 2}^{z} d z^{\prime} \int_{0}^{z^{\prime}} h\left(z^{\prime \prime}\right) d z^{\prime \prime},
$$

which subtracts the value at $L / 2$, so that $g(L / 2)=0$. Alternatively, one can start both integrals at zero and then apply the operator $\{\cdots\}$, as in Eq. (9). For example, $\iint \cos z=\left\{\int \sin z\right\}=\{1-\cos z\}=-\cos z$.

The properties of $\iint$ greatly simplify the operations and the results in Appendix B.

\section{DECOUPLING THE KV EQUATIONS FOR SYMMETRIC MATCHED-BEAM INITIAL CONDITIONS}

For a matched beam with the symmetries of Eqs. (3), we showed in Sec. II that $b(z)=a(z+L)$, implying that Eqs. (1) and (2) are decoupled and that $\langle a\rangle=\langle b\rangle \equiv A$. We write

$$
a(z) \equiv A[1+\rho(z)], \quad b(z) \equiv A[1+\rho(z+L)],
$$

with $\rho(z)>-1$ for all $z$. Then the $Q$ term in Eq. (1) is

$$
\frac{2 Q}{a+b}=\frac{Q}{A}[1-R(h(z))+\cdots],
$$

where $R$ is obtained from an expansion and iterations. All terms consist of integrations of the focus-strength function $h(z)$ [Appendix A]:

$$
F(h(z))=k^{2} \iint\left\{h \int h\right\}+\cdots \equiv k^{2} \delta(z)+\cdots .
$$

Additional terms are shown in the Appendix. With the lattice symmetries of Eqs. (3), all the series terms for $F(z)$, including $\delta(z)$, have only even-numbered cosine harmonics - in contrast to $h(z)$, which has only odd ones.

Equations (1) and (2) are decoupled to all orders so that Eq. (2) is superfluous from here on. Equations (1) and (2) are replaced by

$$
\begin{gathered}
a(z)^{\prime \prime}=-K(z) a+\frac{\in^{2}}{a^{3}}+\frac{Q}{A}\left[1-k^{2} \delta(z)+\cdots\right], \\
b(z)=a(z+L) .
\end{gathered}
$$

\section{EXPANDING AND DECOMPOSING INTO AVERAGE AND PERIODIC PARTS}

Substituting $a=A[1+\rho(z)]$ into Eq. (25), expanding $1 / a^{3}$, dividing by $A$, and using (16) and (24), Eq. (25) is equivalent to

$$
\begin{aligned}
\rho(z)^{\prime \prime}= & -k h(z)-k h(z) \rho+\frac{\alpha}{3}\left(1-3 \rho+6 \rho^{2}-10 \rho^{3}\right. \\
& \left.+15 \rho^{4}-\cdots\right)+q\left[1-k^{2} \delta(z)+\cdots\right] .
\end{aligned}
$$

To solve for the ripple $\rho(z)$ and for the mean radius $A$ (which appears in the definition of $\alpha$ and $q$ ), we decompose Eq. (27) into a pair of equations. Averaging Eq. (27),

$$
\begin{aligned}
0= & -k\langle h \rho\rangle+\frac{\alpha}{3}+2 \alpha\left\langle\rho^{2}\right\rangle-\frac{10}{3} \alpha\left\langle\rho^{3}\right\rangle+5 \alpha\left\langle\rho^{4}\right\rangle \\
& -\cdots+q .
\end{aligned}
$$

Subtracting Eq. (28) from (27),

$$
\begin{aligned}
\rho^{\prime \prime}= & -k h(z)-k\{h \rho\}-\alpha \rho+2 \alpha\left\{\rho^{2}\right\}-\frac{10}{3} \alpha\left\{\rho^{3}\right\} \\
& +5 \alpha\left\{\rho^{4}\right\}-\cdots-q k^{2} \delta(z)+\cdots,
\end{aligned}
$$

with the $\{\cdots\}$ operator defined by Eq. (7). There are now two equations, each containing $\rho(z)$ and $A$-the latter represented by $\alpha$. These are the KV equations (1) and (2) for our matched beam. We will obtain $A$ and $\rho(z)$ to good accuracy in Secs. V, VI, and VII and Appendix E.

\section{ITERATIVE SOLUTION: ARBITRARY SYMMETRIC FOCUSING PROFILE}

On the right-hand side (rhs) of Eq. (29), the $k h(z)$ term dominates the terms involving the unknown function $\rho(z)$. Therefore, we use only $k h(z)$ in the initial integrations which give $\rho_{(0)}$. Then we insert $\rho_{(0)}$ into (29) and integrate again to obtain $\rho_{(1)}$. [A miniscule term, $q k^{2} \delta(z)$, in $\rho_{(0)}$ is omitted.] This process is repeated to get $\rho_{(2)}$ :

$$
\begin{aligned}
& \rho_{(0)}=-k g, \\
& \rho_{(1)}=\rho_{(0)}+\alpha k \iint g+k^{2} \delta+\frac{10}{3} \alpha k^{3} \iint g^{3}, \\
& \rho_{(2)}=\rho_{(1)}-\alpha^{2} k \iiint \int g-k^{3} \iint h \delta-4 \alpha k^{3} \iint g \delta .
\end{aligned}
$$

In (30c), $\iiint \int$ stands for two applications of the operator $\iint$ defined by Eq. (9).

To complete the approximate solution of the KV equations, $\rho(z)$ from Eqs. (30) is put into the matching equation (28). In Eqs. (30) we included no items (e.g., $2 \alpha k^{2} \iint\{g\}^{2}$ ) that would give terms in (28) higher than third power in the parameters $k^{2}, \alpha$, and $q$. That is, we go no higher than third order as defined below.

Some terms vanish by orthogonality, since $h(z), g(z), g^{3}$, etc. possess only odd harmonics while $\delta(z),\left\{g^{2}\right\},\left\{g^{4}\right\}$ have only even ones for symmetric quadrupoles.

A term not shown, $q k^{2} \iint \delta(z)$, involves multiple integrations of an already small function and would contribute $<0.04 \%$ to the maximum radius $a_{\max }$ even at $\sigma_{0}=120^{\circ}$ and affect $A$ by less than 2 parts in 10000 . This is much smaller than the two parts per thousand criterion used for significant terms in Appendix E. 

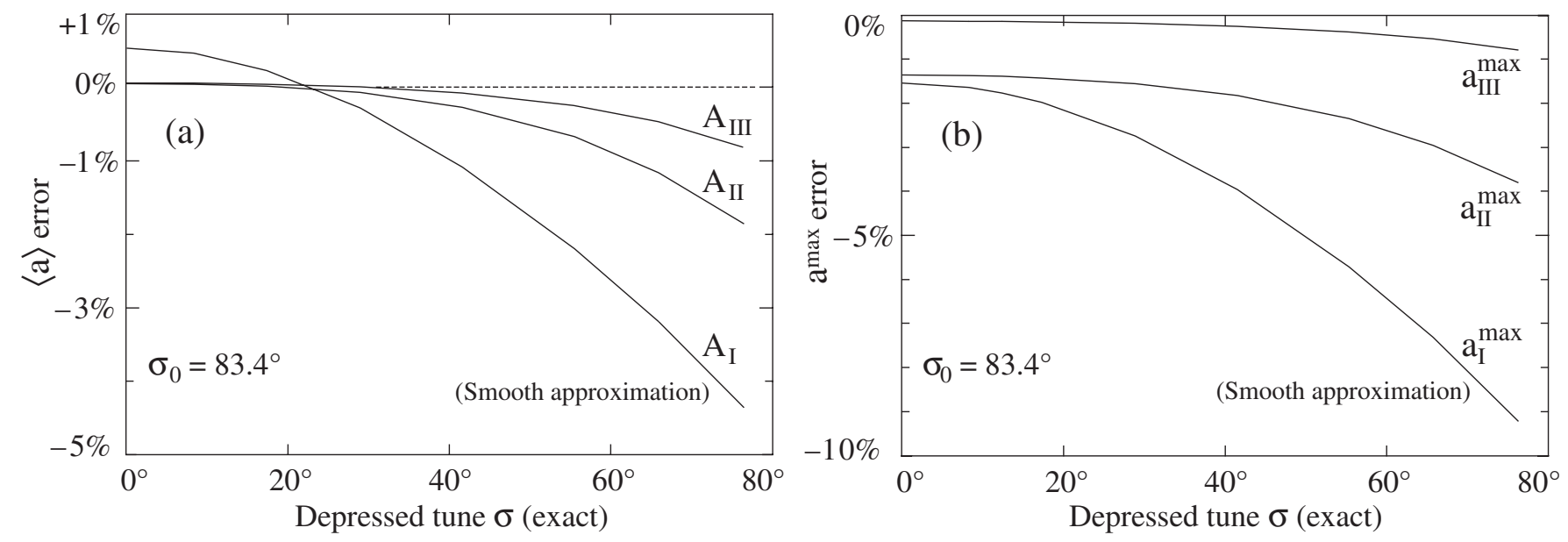

FIG. 1. Illustration of results for special case of FODO lattice, compared with simulation results. (a) Accuracy of mean radius, from Eqs. (34), (36), and (37). (b) Accuracy of maximum radius, from Eqs. (40)-(42). Input quantities: $Q, \in$, and quad voltage $V_{Q}(\propto K)$. Other parameters: see Table II. $V_{Q}$, fixed at $20 \mathrm{kV}$, gives phase advance $\sigma_{0}$ of $83.37^{\circ} ; \in$ and $Q$ are varied so that depressed tune $\sigma$ ranges between $0^{\circ}$ and $76.5^{\circ}$; exact $\sigma_{0}$ and $\sigma$ were obtained numerically.

Order.-We reckon the order of a term in the matching equation by counting the number of factors $k^{2}, \alpha$, and $q$. These would become small parameters in a nondimensional formalism (Appendix J). We prefer to retain physical units for quantities such as the axial coordinate $z$. Note that Lee [8] defines order using $k$ and $\in$ so that our third order corresponds approximately to his fifth order. Appendix B evaluates the combination of Eqs. (28) and (30). The result (simplified in Appendix C) is shown in the next section.

\section{MATCHING EQUATION TO VARIOUS ORDERS FOR AN ARBITRARY SYMMETRIC LATTICE}

\section{A. Third order}

Inserting Eqs. (30) into Eq. (28) yields seven terms [Appendix B, Eq. (B7)]. Some terms combine, resulting in (Appendix C)

$$
K_{\dagger}^{\mathrm{eff}}-\frac{\in_{\mathrm{III}}^{2}}{A_{\mathrm{III}}{ }^{4}}-\frac{Q}{A_{\mathrm{III}}^{2}}=0
$$

where

$$
\begin{aligned}
K_{\dagger}^{\mathrm{eff}} & \equiv\left\langle\left[\int K(z)\right]^{2}\right\rangle\left[1+\frac{1}{24} \Phi\left(1+\frac{20}{27} c_{3}\right)\right] \\
& \in_{\mathrm{III}}{ }^{2} \equiv \in^{2}\left[1+\Phi\left(1+\frac{3}{4} \Phi+3 \beta_{\mathrm{I}}\right)\right] .
\end{aligned}
$$

In Eq. (32), $c_{3}$ is of order unity (see Appendices $\mathrm{G}$ and $\mathrm{H}$ ); in Eq. (33), $\beta_{\mathrm{I}}$ is defined by Eq. (39). Roman-numeral subscripts on $A$ and $\in$ signify the order of approximation - third order in this case. The subscript on $\beta \propto A^{-4}$ indicates that $A_{\mathrm{I}}$ [Eq. (37)] is used to approximate $A$. The matching equation (31) is in the standard form of the smooth approximation, Eq. (37), and can be solved to find the third-order $A$ :

$$
A_{\mathrm{III}}^{2}=\frac{Q}{2 K_{\dagger}^{\text {eff }}}+\left[\left(\frac{Q}{2 K_{\dagger}^{\text {eff }}}\right)^{2}+\frac{\in_{\mathrm{III}}^{2}}{K_{\dagger}^{\text {eff }}}\right]^{1 / 2} .
$$

If the input quantity is the mean radius $A_{\text {inp }}$, then Eq. (31) gives the allowable $Q$ to third order,

$$
Q_{\mathrm{III}}=A_{\mathrm{inp}}^{2} K_{\dagger}^{\mathrm{eff}}-\frac{\in_{\mathrm{III}}^{2}}{A_{\mathrm{inp}}{ }^{2}} \text {. }
$$

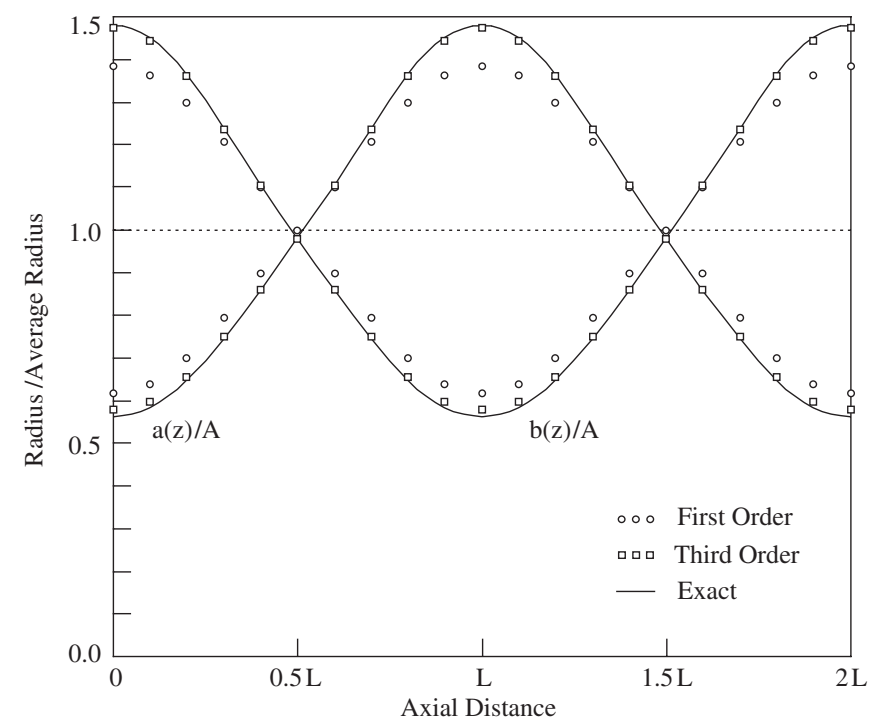

FIG. 2. Illustration of matched envelopes $a(z)$ and $b(z)$ for special case of FODO model. Parameters from Table II, giving tunes $\sigma_{0}=112.2^{\circ} ; \sigma=86.9^{\circ}$. Exact envelopes (solid curves) obtained numerically. Third-order results from Eqs. (30), using Eqs. (E6)-(E10), give $a_{\max }$ error of $-2.37 \%$. Smooth approximation error [Eq. (E6) only] is $-13.0 \%$. Amplitude of halfperiod ripple is $5.6 \%$ of amplitude of full-period ripple. 

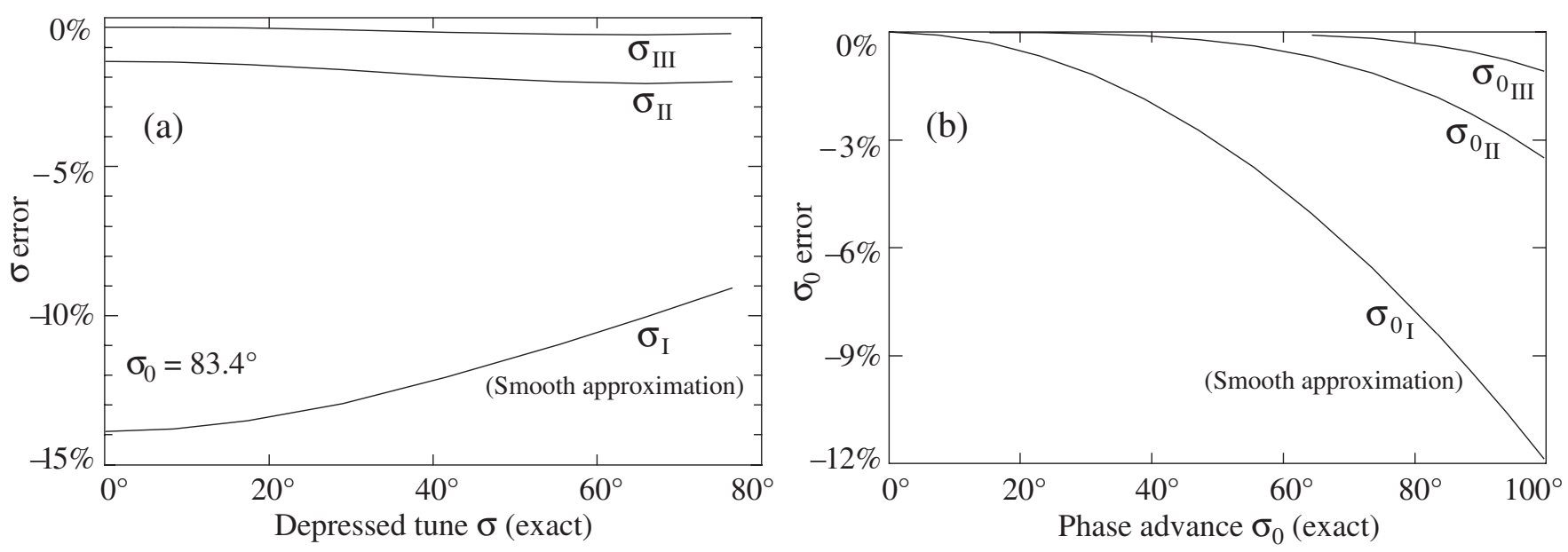

FIG. 3. Phase advances for special case of FODO model with parameters from Table II. (a) Accuracy of depressed tune $\sigma$ from Eqs. (44)-(46). $V_{Q}$ is fixed at $20 \mathrm{kV}$ as in Fig. 1. (b) Accuracy of phase advance $\sigma_{0}$ from Eqs. (47) $-(49)$. $V_{Q}$ ranges from 5 to $22 \mathrm{kV}$.

TABLE II. Parameters for Figs. 1-3, based on the actual MFE ESQ accelerator [12].

\begin{tabular}{lclc}
\hline \hline Quad cell length & $20 \mathrm{~cm}$ & Quad voltage [Figs. 1 and 3(a)] & $20 \mathrm{kV}$ \\
Occupancy factor & 0.5 & Quad voltage (Fig. 2) & $25 \mathrm{kV}$ \\
Quad radius $\left(a_{Q}\right)$ & $1.75 \mathrm{~cm}$ & Beam current (Fig. 2) & $0.5 \mathrm{Amp}$ \\
Beam energy & $200 \mathrm{KeV}$ & Normalized emittance (Fig. 2) & $1.55 \pi \mathrm{mrad}-\mathrm{cm}$ \\
Beam particles & $\mathrm{H}^{-}$ions & & \\
\hline \hline
\end{tabular}

\section{B. Second order}

Equation (B7) has two second-order terms. One yields the correction to $K^{\text {eff }}$ seen in Eq. (32). The other term is $\alpha k^{2}\left\langle g^{2}\right\rangle$, or, using definition Eq. (20), $\alpha \Phi / 3$. We define

$$
\in_{\text {II }}^{2} \equiv \in^{2}(1+\Phi)
$$

and get

$$
K_{\dagger}^{\mathrm{eff}}-\frac{\in_{\mathrm{II}}^{2}}{A_{\mathrm{II}}^{4}}-\frac{Q}{A_{\mathrm{II}}^{2}}=0 .
$$

Equation (36) can be solved for $A_{\mathrm{II}}$ or $Q_{\mathrm{II}}$ in the same way as for the third order, giving useful approximations when $K(z)$ and $\in$ produce $\sigma_{0}$ and $\sigma$ less than about $80^{\circ}$.

\section{First order}

In Eq. (B7), the three terms of lowest order in $\alpha, q, k^{2}$ produce what is called the first-order matching equation in this paper (Ref. [9] used another terminology). This is the classic smooth approximation. These terms give $k^{2}\left\langle\left[\int h\right]^{2}\right\rangle=\alpha / 3+q$, or, using the definitions Eqs. (16), (18), and (19),

$$
K^{\mathrm{eff}}-\frac{\in^{2}}{A_{\mathrm{I}}^{4}}-\frac{Q}{A_{\mathrm{I}}^{2}}=0 .
$$

The equations derived in this paper apply to arbitrary focusing profiles satisfying Eqs. (3), such as the continuous profiles in Appendix G, but for illustration (Figs. 1-3), we use the FODO model (Appendix $\mathrm{H}$ ) with parameters from Table II. First-, second-, and third-order results for $A$, from (34), (36), and (37), are plotted in Fig. 1(a) for FODO. The smooth approximation is seen to be relatively inaccurate except near the point where its error curve crosses the $0 \%$ line.

\section{EXPLICIT THIRD-ORDER RESULT FOR $a^{\text {max }}$}

Knowing the matched mean radius $A$, one can complete the solution for the beam envelope $a(z)=A[1+\rho(z)]$ using $\rho(z)$ from Eqs. (30); $b(z)$ can be found by changing the sign of the terms that contain odd powers of $k$.

Some terms of Eqs. (30) can be written in exact form [Appendix I] for models such as FODO, but Fourier expansion is more useful in general:

$$
h(z)=h_{1}\left[\cos \frac{\pi z}{L}+\frac{c_{3}}{3} \cos \frac{3 \pi z}{L}+\frac{c_{5}}{5} \cos \frac{5 \pi z}{L}+\cdots\right] .
$$

Values (usually of order unity) of $h_{1}$ and $c_{n}$ for both smooth and FODO profiles are given in Appendices G and H. With the definition

$$
\beta_{\mathrm{I}} \equiv \alpha_{\mathrm{I}} \frac{L^{2}}{\pi^{2}}=3 \frac{L^{2}}{\pi^{2}} \frac{\in^{2}}{A_{\mathrm{I}}^{4}}
$$

we have 


$$
\begin{aligned}
a_{\mathrm{III}}^{\max }= & A_{\mathrm{III}}\left[1+\rho_{\mathrm{m}}\left(1+\frac{c_{3}}{27}+\frac{c_{5}}{125}\right)+\frac{1}{8} \rho_{\mathrm{m}}^{2}\left(1+\frac{25 c_{3}}{54}\right)\right. \\
& \left.+\beta_{\mathrm{I}} \rho_{\mathrm{m}}\left(1+\frac{5}{2} \rho_{\mathrm{m}}{ }^{2}+\beta_{\mathrm{I}}\right)\right]
\end{aligned}
$$

using results from Appendix E. The accuracy of Eq. (40) (applied to the special case of the FODO model) is shown in Fig. 1(b), along with that of the truncations

$$
a_{\mathrm{II}}^{\max }=A_{\mathrm{II}}\left[1+\rho_{\mathrm{m}}\left(1+\frac{1}{27} c_{3}+\frac{1}{125} c_{5}\right)+\beta_{\mathrm{I}} \rho_{\mathrm{m}}\right]
$$

and (the smooth approximation)

$$
a_{\mathrm{I}}^{\max }=A_{\mathrm{I}}\left(1+\rho_{\mathrm{m}}\right) .
$$

The $z$-dependence of Eq. (40), from Appendix E, is plotted in Fig. 2.

\section{PHASE ADVANCES: ARBITRARY SYMMETRIC FOCUSING FUNCTIONS}

From the well-known phase-amplitude result [13], the phase advance per quadrupole cell of length $2 L$ is

$$
\sigma=\in \int_{0}^{2 L} \frac{d z}{a^{2}}=2 L \in\left\langle a^{-2}\right\rangle .
$$

We approximate $a(z)$ by $A_{\text {III }}[1+\rho(z)]$ with $A_{\text {III }}$ from Eq. (34) and $\rho(z)$ to third order from Eqs. (30). Subscripts are omitted for brevity. Expanding $a^{-2}$ and taking the average gives

$$
\sigma=2 L \frac{\in}{A_{\mathrm{III}}^{2}}\left[1+3\left\langle\rho^{2}\right\rangle-4\left\langle\rho^{3}\right\rangle+5\left\langle\rho^{4}\right\rangle-\cdots\right] .
$$

(The $2 \rho$ term has zero average by definition.) Appendix D shows that to third-order accuracy

$$
\sigma_{\mathrm{III}}=2 L \frac{\in}{A_{\mathrm{III}}^{2}}\left[1+\Phi\left(1+\frac{3}{4} \Phi+2 \beta_{\mathrm{I}}\right)\right]
$$

for arbitrary symmetric AG lattice functions. Errors with respect to exact values from simulations are illustrated in Fig. 3(a) for the particular case of a FODO lattice. Useful accuracy is retained after dropping two terms and using lower-order $A_{\text {II }}$ from Eq. (36):

$$
\sigma_{\mathrm{II}}=2 L \frac{\epsilon}{A_{\mathrm{II}}^{2}}(1+\Phi) .
$$

Figure 3(a) shows large errors for the first-order result (smooth approximation):

$$
\sigma_{\mathrm{I}}=2 L \frac{\epsilon}{A_{\mathrm{I}}^{2}} .
$$

The undepressed tune $\sigma_{0}$ for arbitrary symmetric focusing profiles is found by setting $Q=0$ in Eq. (31), then eliminating $\in$ from Eq. (44). Details are in Appendix D. The third-order result is (cf. Sec. IX)

$$
\sigma_{0 \mathrm{III}}=2 L\left(K_{\dagger}^{\mathrm{eff}}\right)^{1 / 2}\left[1+\frac{1}{2} \Phi+\frac{3}{4} \Phi^{2}\right]
$$

for arbitrary symmetric focusing functions. To second order,

$$
\sigma_{0 \mathrm{II}}=2 L\left(K_{\dagger}^{\mathrm{eff}}\right)^{1 / 2}\left[1+\frac{1}{2} \Phi\right] .
$$

The smooth approximation is

$$
\sigma_{0 \text { I }}=2 L\left(K^{\mathrm{eff}}\right)^{1 / 2} .
$$

Figure 3 compares the accuracy of Eqs. (47)-(49) for the special case of FODO focusing (with occupancy $\eta=0.5$ ), but gives an idea of the relative accuracy for arbitrary cases. At $\sigma_{0}=100^{\circ}$, the smooth approximation is off by $12 \%$; this is improved to $1.4 \%$ for third order.

\section{UNDEPRESSED TUNE: A SIMPLE, ACCURATE APPROXIMATION}

For the symmetric FODO model, $\sigma_{0}$, obtained by multiplying transfer matrices [2], is given exactly by

$$
\begin{aligned}
\sigma_{0}= & \cos ^{-1}[\cos \kappa \cosh \kappa \\
& +(1-\eta) K^{1 / 2} L(\cos \kappa \sinh \kappa-\sin \kappa \cosh \kappa) \\
& \left.-\frac{1}{2}(1-\eta)^{2} K L^{2}(\sin \kappa \sinh \kappa)\right],
\end{aligned}
$$

where $\kappa=\eta k^{1 / 2} L$. Equation (50) applies to cases where $\sigma_{0}<180^{\circ}$.

This exact solution can be expanded to give the accurate approximation

$$
\sigma_{0} \sim 2 \sin ^{-1}\left[L\left(K^{\mathrm{eff}}\right)^{1 / 2}\right]
$$

where for FODO, $\quad K^{\text {eff }}=\frac{1}{12} \eta^{2}(3-2 \eta) k^{2} L^{2}$. Equation (51) resembles Eq. (49), the smooth approximation, but (for $\eta=0.5, \sigma_{0}=100^{\circ}$ ) the error is reduced from $12 \%$ to $0.41 \%$. This is actually better accuracy than given by Eq. (47) (see Fig. 3).

For non-FODO cases, such as the examples in Appendix G, $K^{\text {eff }}$ in Eq. (51) would be calculated from Eq. (19) or (F10). It would be interesting to compare the accuracies of Eqs. (47) and (51) for such cases.

\section{WORK FOR THE FUTURE}

It would be useful to investigate the modifications that would be required to extend the methods of this paper to nonsymmetric AG profiles, which are often used in practice.

\section{ACKNOWLEDGMENTS}

I thank E. P. Lee and S. M. Lund for helpful discussions. Special thanks to L. L. LoDestro for her contributions, including major improvements to Secs. II and III. This work was supported in part by the U.S. Department of Energy under Contract No. DE-AC03-76SF00098. 


\section{APPENDIX A: DECOUPLING $a$ AND $b$ IN THE KV EQUATIONS}

This Appendix calculates $a(z)+b(z)$ for the $Q$-term denominators in the KV equations. Putting Eq. (15) into Eq. (1) and expanding gives

$$
\begin{gathered}
\rho(z)^{\prime \prime}=-k h(z)-k h(z) \rho+\frac{\in^{2}}{A^{4}}(1-3 \rho+\cdots) \\
+\frac{Q}{A^{2}}\left[1-R(z)+R^{2}(z)-\cdots\right],
\end{gathered}
$$

where, using Eq. (5),

$$
R(z) \equiv \frac{\rho(z)+\rho(z+L)}{2} .
$$

We will find that only lowest-order results are needed and will drop the $R^{2}$ term, etc. We subtract the average of Eq. (A1), using definitions from Table I, and obtain

$$
\rho^{\prime \prime}=-k h(z)-k\{h \rho\}-\alpha \rho-q R(z)+\cdots,
$$

which we solve by iteration. In the initial step, the lowestorder quantity is $k h(z)$, so that

$$
\rho_{(0)}^{\prime \prime}=-k h, \quad \rho_{(0)}=-k g .
$$

Substituting $-k g$ for $\rho$ in the previous equation,

$$
\begin{gathered}
\rho_{(1)}^{\prime \prime}=-k h+k^{2}\{h g\}+\alpha k g+\frac{q k}{2}[g(z)+g(z+L)] ; \\
\rho_{(1)}(z)=-k g+k^{2} \delta+\alpha k \iint g+\frac{q k}{2} \iint[g(z)+g(z+L)],
\end{gathered}
$$

where we used the definition of $\delta$. Using the shift Eq. (3c),

$$
\begin{aligned}
\rho_{(1)}(z+L)= & +k g+k^{2} \delta-\alpha k \iint g \\
& -\frac{q k}{2}[g(z)+g(z+L)] .
\end{aligned}
$$

Then Eqs. (A2), (A6), and (A7) give

$$
R(z)=k^{2} \delta+\cdots,
$$

resulting in Eq. (25) in Sec. III.

The expansion and iteration could be extended to produce more terms [all derived solely from $h(z)$ ] but these would give even smaller corrections to our results.

\section{APPENDIX B: DETAILS OF DERIVATION OF THE MATCHING EQUATION}

Rearranging Eq. (28) slightly gives

$$
k\langle h \rho\rangle=\alpha\left[\frac{1}{3}+2\left\langle\rho^{2}\right\rangle-\frac{10}{3}\left\langle\rho^{3}\right\rangle+5\left\langle\rho^{4}\right\rangle+\cdots\right]+q .
$$

1. Left-hand side (lhs) of Eq. (B1)

Inserting $\rho$ from Eqs. (30), the lhs of this equation is

$$
\begin{aligned}
k\langle h \rho\rangle= & k^{2}\left[-\langle h g\rangle+\alpha\left\langle h \iint g\right\rangle+\frac{10}{3} \alpha k^{2}\left\langle h \iint g^{3}\right\rangle\right. \\
& -\alpha^{2}\left\langle h \iiint \int g\right\rangle-k^{2}\left\langle h \iint h \delta\right\rangle-4 \alpha k^{2}\left\langle h \iint g \delta\right\rangle \\
& +\cdots]
\end{aligned}
$$

where we have dropped the subscripts on $\rho$. The orthogonal $k^{2} \delta$ term is absent. We rearrange the integrations, noting that $\left\langle u \int v\right\rangle=-\left\langle v \iint u\right\rangle$ if $\langle u\rangle\langle v\rangle=0$ and that $\left\langle u \iint v\right\rangle=+\left\langle v \iint u\right\rangle$ if $\langle u\rangle=\langle v\rangle=0$. For example, $-\left\langle h \iiint \int g\right\rangle=-\left\langle g \iint g\right\rangle=+\left\langle\left[\int g\right]^{2}\right\rangle$. Applying this technique throughout gives

$$
\begin{aligned}
l h s= & +k^{2}\left[\left\langle\left[\int h\right]^{2}\right\rangle+\alpha\left\langle g^{2}\right\rangle+\alpha^{2}\left\langle\left[\int g\right]^{2}\right\rangle\right] \\
& +k^{4}\left[\left\langle h \iint h \delta\right\rangle+\frac{10}{3} \alpha\left\langle g^{4}\right\rangle-4 \alpha\left\langle g^{2} \delta\right\rangle+\cdots\right] .
\end{aligned}
$$

and will allow us to eliminate four terms in Eq. (B7).

\section{Right-hand side (rhs) of Eq. (B1)}

For the rhs of (B1), the significant terms are

$$
\begin{gathered}
\left\langle\rho^{2}\right\rangle=k^{2}\left\langle g^{2}\right\rangle-2 \alpha k^{2}\left\langle g \iint g\right\rangle+2 k^{4}\left\langle g \iint h \delta\right\rangle+\cdots, \\
\left\langle\rho^{3}\right\rangle=3 k^{4}\left\langle g^{2} \delta\right\rangle+\cdots, \\
\left\langle\rho^{4}\right\rangle=k^{4}\left\langle g^{4}\right\rangle+\cdots
\end{gathered}
$$

The very small $k^{4}\left\langle\delta^{2}\right\rangle$ term was omitted from $\left\langle\rho^{2}\right\rangle$. Again changing the order of integrations, the rhs of (B1) becomes

$$
\begin{aligned}
\operatorname{rhs}= & \alpha\left[\frac{1}{3}+k^{2}\left[2\left\langle g^{2}\right\rangle+4 \alpha\left\langle\left[\int g\right]^{2}\right\rangle\right]\right. \\
& \left.+k^{4}\left[4\left\langle g \iint h \delta\right\rangle-10\left\langle g^{2} \delta\right\rangle+5\left\langle g^{4}\right\rangle\right]+\cdots\right]+q .
\end{aligned}
$$

Four of the terms of (B6) combine with terms of (B2), so that

$$
\begin{aligned}
k^{2}\left\langle\left[\int h\right]^{2}\right\rangle+k^{4}\left\langle h \iint h \delta\right\rangle= & \alpha\left[\frac{1}{3}+k^{2}\left(\left\langle g^{2}\right\rangle+3 \alpha k^{2}\left\langle\left[\int g\right]^{2}\right\rangle\right)\right. \\
& +k^{4}\left(4\left\langle g \iint h \delta\right\rangle-6\left\langle g^{2} \delta\right\rangle\right. \\
& \left.\left.+\frac{5}{3}\left\langle g^{4}\right\rangle\right)+\cdots\right]+q,
\end{aligned}
$$

the matching equation from Eq. (B1). Each term (except $\alpha / 3$ and $q$ ) involves averages of functions of the focusing 
TABLE III. $\quad h_{1}, c_{n} \equiv n h_{n} / h_{1}$, and $\eta_{\text {equiv }}$ for four smooth models.

\begin{tabular}{lccccccc}
\hline \hline Model & $h_{1}$ & $c_{1}$ & $c_{3}$ & $c_{5}$ & $c_{7}$ & $c_{9}$ & $\eta_{\text {equiv }}$ \\
\hline$\# 1$ & 1 & 1 & 0 & 0 & 0 & 0 & 0.57 \\
$\# 2$ & $9 / 8$ & 1 & $-1 / 3$ & 0 & 0 & 0 & 0.69 \\
$\# 3$ & $3 / 4$ & 1 & 1 & 0 & 0 & 0 & 0.40 \\
$\# 4$ & $15 / 16$ & 1 & $1 / 2$ & $-1 / 2$ & 0 & 0 & 0.53 \\
\hline \hline
\end{tabular}

TABLE IV. $\quad h_{1}$ and $c_{n} \equiv n h_{n} / h_{1}$ for FODO case with four different occupancies.

\begin{tabular}{lccccccc}
\hline \hline$\eta$ & $\pi h_{1} / 2$ & $c_{1}$ & $c_{3}$ & $c_{5}$ & $c_{7}$ & $c_{9}$ & $c_{11}$ \\
\hline $1 / 3$ & 1 & 1 & 2 & 1 & -1 & -2 & -1 \\
$1 / 2$ & $\sqrt{2}$ & 1 & 1 & -1 & -1 & 1 & 1 \\
$2 / 3$ & $\sqrt{3}$ & 1 & 0 & -1 & 1 & 0 & -1 \\
1 & 2 & 1 & -1 & 1 & -1 & 1 & -1 \\
\hline \hline
\end{tabular}

profile $h(z)$. Given any $h(z)$ _obtained from a model such as FODO or measured on an actual quadrupole cell - these averages can be calculated once and for all, being constant coefficients of the terms in $\alpha$ and $k$. Appendix $\mathrm{C}$ shows how to write Eq. (B7) in simple form [Eq. (C10)].

\section{APPENDIX C: SIMPLIFICATION OF THE MATCHING EQUATION}

It is convenient to write the Fourier representation in the form

$$
h(z)=h_{1}\left[\cos \frac{\pi z}{L}+\frac{1}{3} c_{3} \cos 3 \frac{\pi z}{L}+\frac{1}{5} c_{5} \cos 5 \frac{\pi z}{L} \cdots\right]
$$

The axial profile of the quadrupole gradient determines $h_{1}$ and $c_{n}$. Tables III and IV show that $h_{1}$ remains of the order of unity while $c_{3}$ and $c_{5}$ can change sign as the profile is varied. For the hard-edge quadrupole model (FODO) with occupancy $\eta=0.5$, Table IV shows that $c_{3}=1$. Because of multiple integrations, terms containing $c_{5}$ are usually negligible.

\section{Right-hand side of Eq. (B7)}

By definition, $k^{2}\left\langle g^{2}\right\rangle=\Phi / 3$. For the factor $\left\langle\left[\int g\right]^{2}\right\rangle=$ $\left\langle\left[\iiint h\right]^{2}\right\rangle$, the third and higher harmonics make very small $(0.1 \%)$ contributions because of the multiple integrations. Comparing leading terms for $\left\langle\left[\int g\right]^{2}\right\rangle$ and $\left\langle g^{2}\right\rangle$ gives

$$
3 k^{2}\left\langle\left[\int g\right]^{2}\right\rangle \approx \frac{L^{2}}{\pi^{2}} 3 k^{2}\left\langle g^{2}\right\rangle=\frac{L^{2}}{\pi^{2}} \Phi .
$$

The three $k^{4}$ terms on the rhs of Eq. (B7) are

$$
\begin{array}{r}
4\left\langle g \iint h \delta\right\rangle=\frac{1}{2}\left\langle g^{2}\right\rangle^{2}\left(1+\frac{19}{27} c_{3}+\cdots\right), \\
-6\left\langle g^{2} \delta\right\rangle=-\frac{3}{4}\left\langle g^{2}\right\rangle^{2}\left(1+\frac{4}{9} c_{3}+\cdots\right), \\
\frac{5}{3}\left\langle g^{4}\right\rangle=\frac{5}{2}\left\langle g^{2}\right\rangle^{2}\left(1+\frac{4}{81} c_{3}+\cdots\right) .
\end{array}
$$

Adding the rhs's of Eqs. (C3)-(C5) gives $\frac{3}{4}\left\langle g^{2}\right\rangle^{2}[1+$ $\left.0.063 c_{3}+\cdots\right]$, where the small $c_{3}$ correction can be neglected since it corrects a term which is already third order. Using all these results along with definition (16), the quantity in brackets on the rhs of Eq. (B7) is

$$
\begin{aligned}
& \in^{2}\left(1+\Phi+\frac{3}{4} \Phi^{2}+\frac{9}{\pi^{2}} \frac{\in^{2} L^{2}}{A_{\mathrm{I}}^{4}} \Phi\right) ; \\
& \in_{\mathrm{III}}{ }^{2} \equiv \in^{2}\left(1+\Phi+\frac{3}{4} \Phi^{2}+3 \beta_{\mathrm{I}} \Phi\right),
\end{aligned}
$$

which is Eq. (33). In the last term,

$$
\beta_{\mathrm{I}} \equiv 3 \frac{L^{2}}{\pi^{2}} \frac{\in^{2}}{A_{\mathrm{I}}^{4}},
$$

uses the lowest-order value for $A$ because this term is already of the highest order that we retain.

\section{Left-hand side of Eq. (B7)}

The smaller term is

$$
\left\langle h \iint h \delta\right\rangle=\frac{1}{8}\left\langle g^{2}\right\rangle\left(1+\frac{20}{27} c_{3}+\cdots\right)\left\langle\left[\int h\right]^{2}\right\rangle .
$$

We define the lhs of (B7) as

$$
K_{\dagger}^{\mathrm{eff}} \equiv k^{2}\left\langle\left[\int h\right]^{2}\right\rangle\left[1+\frac{1}{24} \Phi\left(1+\frac{20}{27} c_{3}\right)\right]
$$

i.e., Eq. (32). Altogether,

$$
K_{\dagger}^{\mathrm{eff}}=\frac{\in_{\mathrm{III}}^{2}}{A_{\mathrm{III}}^{4}}+\frac{Q}{A_{\mathrm{III}}^{2}},
$$

which is Eq. (31).

\section{APPENDIX D: DEPRESSED AND UNDEPRESSED TUNES, GENERAL CASE}

\section{Depressed tune}

Here we evaluate the expansion terms in Eq. (43). From Eqs. (B3)-(B5) in Appendix B,

$$
3\left\langle\rho^{2}\right\rangle=3 k^{2}\left\langle g^{2}\right\rangle+6 \alpha k^{2}\left\langle\left[\int g\right]^{2}\right\rangle+6 k^{4}\left\langle g \iint h \delta\right\rangle+\cdots,
$$

$$
\begin{aligned}
-4\left\langle\rho^{3}\right\rangle & =-12 k^{4}\left\langle g^{2} \delta\right\rangle+\cdots, \\
5\left\langle\rho^{4}\right\rangle & =5 k^{4}\left\langle g^{4}\right\rangle+\cdots .
\end{aligned}
$$


From Appendix C, Eqs. (C2)-(C5),

$$
\begin{gathered}
6 \alpha k^{2}\left\langle\left[\int g\right]^{2}\right\rangle \approx \frac{L^{2}}{\pi^{2}} 6 \alpha k^{2}\left\langle g^{2}\right\rangle=2 \beta_{\mathrm{I}} \Phi, \\
6 k^{4}\left\langle g \iint h \delta\right\rangle=\frac{3}{4} k^{4}\left\langle g^{2}\right\rangle^{2}\left(1+\frac{19}{27} c_{3}+\cdots\right), \\
-12 k^{4}\left\langle g^{2} \delta\right\rangle=-\frac{3}{2} k^{4}\left\langle g^{2}\right\rangle\left(1+\frac{4}{9} c_{3}+\cdots\right), \\
5 k^{4}\left\langle g^{4}\right\rangle=\frac{15}{2} k^{4}\left\langle g^{2}\right\rangle^{2}\left(1+\frac{4}{81} c_{3}+\cdots\right) .
\end{gathered}
$$

When Eqs. (D5)-(D7) are added, the $c_{3}$ coefficient is only 25/729. Dropping this and using the definitions gives, to third-order accuracy

$$
\sigma=2 L \frac{\in}{A_{\mathrm{III}}^{2}}\left(1+\Phi+\frac{3}{4} \Phi^{2}+2 \beta_{\mathrm{I}} \Phi\right) .
$$

\section{Undepressed tune}

Setting $Q=0$, Eq. (31) is

$$
K_{\dagger}^{\mathrm{eff}}=\frac{\in_{\mathrm{III}}{ }^{2}}{A_{\mathrm{III}}{ }^{4}}=\frac{\in^{2}}{A_{\mathrm{III}}{ }^{4}}\left(1+\Phi+\frac{3}{4} \Phi^{2}+3 \beta_{\mathrm{I}} \Phi\right)^{1 / 2} \text {. }
$$

The factor $\in^{2} / A_{\mathrm{I}}^{4}$ in $\beta_{\mathrm{I}}$ can be replaced by $K^{\mathrm{eff}} \equiv$ $k^{2}\left\langle\left[\int h\right]^{2}\right\rangle$, according to Eq. (37) with $Q=0$. Comparing with the definition of $\Phi$ and Fourier expanding as before, the last term, to lowest order, is $3 \Phi^{2}$ for $Q=0$. Thus, altogether,

$$
K_{\dagger}^{\mathrm{eff}}=\frac{\in^{2}}{A_{\mathrm{III}}^{4}}\left(1+\Phi+\frac{7}{2} \Phi^{2}+\cdots\right)^{1 / 2} .
$$

Making a similar replacement in Eq. (D8) with $Q=0$,

$$
\sigma_{0}=2 L \frac{\in}{A_{\mathrm{III}}^{2}}\left(1+\Phi+\frac{11}{4} \Phi^{2}+\cdots\right) .
$$

Using Eq. (D10) to eliminate $\in / A_{\mathrm{III}}^{2}$,

$$
\begin{aligned}
\sigma_{0}= & 2 L\left(K_{\dagger}^{\mathrm{eff}}\right)^{1 / 2}\left(1+\Phi+\frac{11}{4} \Phi^{2}+\cdots\right) \\
& \times\left(1+\Phi+\frac{15}{4} \Phi^{2}+\cdots\right)^{-1 / 2},
\end{aligned}
$$

or, finally, to third order,

$$
\sigma_{0}=2 L\left(K_{\dagger}^{\mathrm{eff}}\right)^{1 / 2}\left(1+\frac{1}{2} \Phi+\frac{3}{4} \Phi^{2}+\cdots\right)
$$

for arbitrary symmetric AG profiles. Sometimes it is convenient to work with the squares of $\sigma_{0}$ and $\sigma$, which are, for third order

$$
\sigma_{0}^{2}=4 L^{2} K_{\dagger}^{\mathrm{eff}}\left(1+\Phi+\frac{7}{4} 2 \Phi^{2}\right)
$$

and

$$
\sigma^{2}=4 L^{2} \frac{\in^{2}}{A_{\mathrm{III}}^{4}}\left[1+2 \Phi\left(1+\frac{5}{4} \Phi+2 \beta_{\mathrm{I}}\right)\right] .
$$

\section{APPENDIX E: CALCULATION OF $a(z)$ AND $b(z)$ FOR THE GENERAL CASE}

Using Fourier expansion, written as in Appendix C,

$k h(z)=k h_{1}\left[\cos \frac{\pi z}{L}+\frac{1}{3} c_{3} \cos 3 \frac{\pi z}{L}+\frac{1}{5} c_{5} \cos 5 \frac{\pi z}{L} \cdots\right]$, and recalling $\rho_{\mathrm{m}} \equiv h_{1} k L^{2} / \pi^{2}$, the terms of Eqs. (30) are

$$
\begin{aligned}
-k g(z)= & -k \iint h=\rho_{m}\left[\cos \frac{\pi z}{L}+\frac{1}{27} c_{3} \cos 3 \frac{\pi z}{L}\right. \\
& \left.+\frac{1}{125} c_{5} \cos 5 \frac{\pi z}{L}+\cdots\right], \\
\alpha k \iint g= & \beta \rho_{m}\left[\cos \frac{\pi z}{L}+\frac{1}{243} c_{3} \cos 3 \frac{\pi z}{L}+\cdots\right], \\
k^{2} \delta(z)= & k^{2} \iint\{h g\}=\frac{1}{8} \rho_{m}^{2}\left[\left(1+\frac{10}{27} c_{3} \cdots\right) \cos 2 \frac{\pi z}{L}\right. \\
& \left.+\frac{5}{54} c_{3} \cos 4 \frac{\pi z}{L}+\cdots\right],
\end{aligned}
$$

$$
\begin{aligned}
\frac{10}{3} \alpha k^{3} \iint g^{3}= & \frac{5}{2} \beta \rho_{m}{ }^{3}\left[\left(1+\frac{1}{27} c_{3}+\cdots\right) \cos \frac{\pi z}{L}\right. \\
& \left.+\frac{1}{27}\left(1+\frac{2}{9} c_{3}+\cdots\right) \cos 3 \frac{\pi z}{L}+\cdots\right], \\
- & \alpha^{2} k \iiint \int g=\beta^{2} \rho_{m} \cos \frac{\pi z}{L}+\cdots .
\end{aligned}
$$

The small final two terms from Eqs. (30) have been omitted here for simplicity.

\section{The significant terms}

We drop small quantities in the above equations. The criterion is that they contribute less than two parts per thousand to the final result for a bad-case scenario: large focusing strength (giving phase advance of $112^{\circ}$ ) and large $\beta$. This leaves

$$
\begin{gathered}
-k g=\rho_{m}\left[\cos \frac{\pi z}{L}+\frac{1}{27} c_{3} \cos 3 \frac{\pi z}{L}\right. \\
\left.+\frac{1}{125} c_{5} \cos 5 \frac{\pi z}{L}+\cdots\right], \\
\alpha k \iint g=\beta \rho_{m} \cos \pi \frac{\pi z}{L}+\cdots,
\end{gathered}
$$

$$
k^{2} \delta(z)=\frac{1}{8} \rho_{m}{ }^{2}\left[\cos 2 \frac{\pi z}{L}+\frac{25}{54} c_{3} \cos 4 \frac{\pi z}{L}+\cdots\right]
$$




$$
\begin{array}{r}
\frac{10}{3} \alpha k^{3} \iint g^{3}=\frac{5}{2} \beta \rho_{m}{ }^{3} \cos \frac{\pi z}{L}, \\
-\alpha^{2} k \iiint \int g=\beta^{2} \rho_{m} \cos \frac{\pi z}{L}+\cdots .
\end{array}
$$

Adding all these gives $\rho(z)$ to third-order accuracy. A few small terms were omitted as mentioned before. Setting $z=0$ gives $\rho_{\max }$ and $a_{\max }=A\left(1+\rho_{\max }\right)$ as presented in Sec. VII. Setting $z=L$ changes the sign of all terms, except the even term $k^{2} \delta(z)$, and yields $a_{\min } . \alpha$ and $\beta$ are only needed to lowest order, i.e., $\alpha_{\mathrm{I}}$ and $\beta_{\mathrm{I}}$; we omit the subscripts here.

Results from Eqs. (E6)-(E10) are shown in Fig. 2 in the main text.

\section{APPENDIX F: FOURIER REPRESENTATION, ARBITRARY SYMMETRIC CASES}

\section{Fourier coefficients}

Recall from Sec. II that the focusing force $K(z)$ in the $\mathrm{KV}$ equations is written as

$$
K(z)=k h(z)
$$

with $h(0)=1$. Because of the symmetries [Eqs. (3)] and initial conditions, there are only odd harmonics and no sine terms:

$$
h(z)=\sum_{1,3,5, \ldots} h_{n} \cos \frac{n \pi z}{L}
$$

with the condition

$$
\sum_{1,3,5, \ldots} h_{n}=1
$$

The Fourier coefficients are

$$
h_{n}=\frac{1}{L} \int_{0}^{2 L} h(z) \cos \frac{n \pi z}{L} d z .
$$

It is often convenient to define

$$
c_{n} \equiv n \frac{h_{n}}{h_{1}}
$$

where $c_{1}=1$ by definition and where $\left|c_{3}\right|$ usually turns out to be of order unity - see Tables III and IV. Then Eq. (F2) is written as

$$
h(z)=h_{1} \sum_{1,3,5, \ldots} \frac{1}{n} c_{n} \cos \frac{n \pi z}{L} .
$$

\section{Solution of envelope equation}

In the solution for $\rho(z)$, Eqs. (30), the largest term is

$$
\rho_{(0)}(z)=-k g(z)=-k \int_{L / 2}^{z} d z^{\prime} \int_{o}^{z^{\prime}} h\left(z^{\prime \prime}\right) d z^{\prime \prime},
$$

which with Eqs. (F2) and (F5) is

$$
-k g(z)=\frac{k L^{2}}{\pi^{2}} h_{1} \sum_{1,3,5, \ldots} \frac{c_{n}}{n^{3}} \cos \frac{n \pi z}{L} .
$$

The next largest term is

$$
\alpha k \iint g=\alpha \frac{k L^{4}}{\pi^{4}} h_{1} \sum_{1,3,5, \ldots} \frac{c_{n}}{n^{5}} \cos \frac{n \pi z}{L} .
$$

To achieve $1 \%$ accuracy, the first three series elements of (F8) are usually required, whereas for Eq. (F9), only the fundamental is needed [cf. Eq. (E2)].

The additional terms of Eqs. (30), shown in Eqs. (E3) and (E4), are found with the help of trigonometric identities.

The mean square of the integral of Eq. (F2) gives the effective force

$$
K^{\mathrm{eff}} \equiv k^{2}\left\langle\left[\int h\right]^{2}\right\rangle=h_{1}{ }^{2} \frac{k^{2} L^{2}}{2 \pi^{2}} \sum_{1,3,5, \ldots} \frac{c_{n}{ }^{2}}{n^{4}},
$$

which is used in the matching equation and for calculating the undepressed phase advance.

The correction term $\Phi$ (used in evaluating phase advances, average radius or transportable current, etc.) is

$$
\Phi \equiv 3 k^{2}\left\langle g^{2}\right\rangle=3 h_{1}{ }^{2} \frac{k^{2} L^{4}}{2 \pi^{4}} \sum_{1,3,5, \ldots} \frac{c_{n}{ }^{2}}{n^{6}} \rightarrow 3 h_{1}{ }^{2} \frac{k^{2} L^{4}}{2 \pi^{4}}
$$

since the harmonics contribute practically nothing. Dividing by Eq. (F10), we find

$$
\Phi=3 K^{\mathrm{eff}} \frac{L^{2}}{\pi^{2}}\left(1-\frac{c_{3}^{2}}{81}+\cdots\right),
$$

which could be useful in certain calculations.

\section{APPENDIX G: SOLUTION FOR SOME SMOOTH LATTICE PROFILES}

All our results apply to an arbitrary symmetric focusing profile once its Fourier coefficients are known. Some simple but representative smooth models are discussed here. None of our examples require more than two harmonics.

Field model 1. $-h(z)=\cos \frac{\pi z}{L}$.

$$
h_{1}=1, \quad c_{3}=0 .
$$

This is the model used by Lee [8].

Field model 2. $-K^{\prime \prime}(0)=0$.

$$
h_{1}=\frac{9}{8}, \quad c_{3}=-\frac{1}{3},
$$

with all the other coefficients zero except $c_{1}$, which is unity by definition. This choice gives a flat field at the midpoint of the quadrupoles, without the discontinuities of the hardedge FODO model. From Eqs. (G1) and (F10) we get

$$
K^{\mathrm{eff}}=\frac{9^{2}}{8^{2}} \frac{k^{2} L^{2}}{2 \pi^{2}}\left(1+\frac{1}{9^{3}}\right)
$$




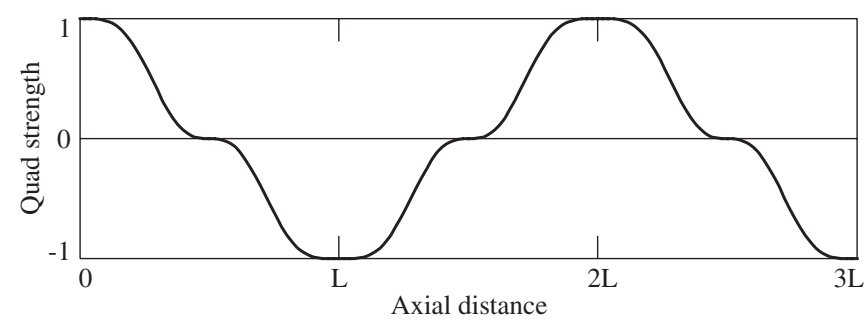

FIG. 4. Axial profile of normalized quadrupole strength $h(z)$ for smooth field model \#4, which uses only the 3rd and 5th harmonics. The unit cell length is $2 L$.

$$
\Phi=3 \frac{9^{2}}{8^{2}} \frac{k^{2} L^{4}}{2 \pi^{4}}\left(1+\frac{1}{9^{4}}\right)
$$

The $1 / 9^{4}$ term can be neglected in practice.

Field model 3. $-K^{\prime}(L / 2)=0$.

$$
h_{1}=\frac{3}{4}, \quad c_{3}=1 .
$$

This model is narrow, peaked at the quadrupole midpoints, with zero slope at the gap centers. It gives focusing strength equivalent to FODO [Eq. (I1)] having about $40 \%$ occupancy. The third-harmonic corrections to $K^{\mathrm{eff}}$ and $\Phi$ are $1 / 81$ and $1 / 243$, respectively.

Field model 4. $-K^{\prime \prime}(0)=0$ and $K^{\prime}(L / 2)=0$.

$$
h_{1}=\frac{15}{16}, \quad c_{3}=\frac{1}{2}, \quad c_{5}=-\frac{1}{2},
$$

which gives a fairly realistic profile (Fig. 4) and corresponds to FODO with $\eta \sim 53 \%$. The third- and fifthharmonic corrections are well under $1 \%$ for this case.

Table III summarizes the above results.

\section{APPENDIX H: FOURIER SOLUTION FOR THE SPECIAL CASE OF FODO}

In the case of the popular FODO lattice model (Fig. 5), the Fourier coefficients $h_{n}$ are readily calculated from Eq. (F4):

$$
h_{n}=\frac{4}{n \pi} \sin \frac{n \pi \eta}{2},
$$

which satisfies Eq. (F3) for occupancy $\eta$ over the range $0<\eta \leq 1$. From Eq. (F5),

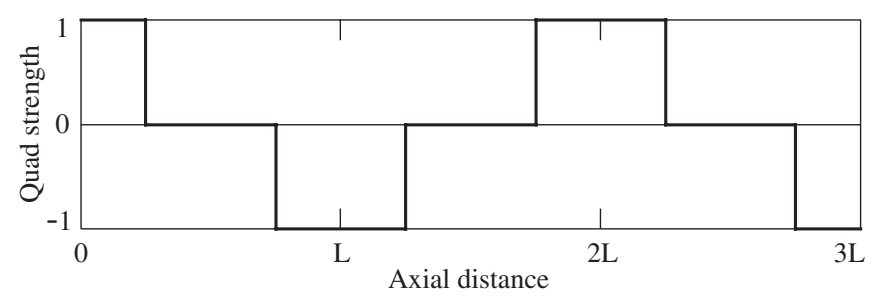

FIG. 5. Normalized quadrupole strength $h(z)$ vs $z$ for a FODO lattice having occupancy 0.5 . The unit cell length is $2 L$.

$$
c_{n}=n \frac{\sin (n \pi \eta / 2)}{\sin (\pi \eta / 2)}
$$

for FODO. All the results from Appendix F can be used for FODO by putting $h_{1}=\sin \frac{\pi \eta}{2}$. Values of $h_{1}$ (normalized with $\pi / 2$ ) and $c_{n}$ are shown in Table IV for various $\eta$.

\section{APPENDIX I: SOME EXACT FORMULAS FOR THE SPECIAL CASE OF FODO}

Results using truncated Fourier representations for the hard-edge FODO may be compared with exact results by integration. (The FODO model is illustrated in Fig. 5.) Because of the symmetries expressed in Eqs. (3), the calculation of averages is simplified, requiring only integration over one-fourth of a cell. One finds for a specified occupancy $\eta$

$$
\begin{gathered}
K^{\mathrm{eff}} \equiv k^{2}\left\langle\left[\int h\right]^{2}\right\rangle=\frac{1}{12} \eta^{2}(3-2 \eta) k^{2} L^{2}, \\
\Phi \equiv 3 k^{2}\left\langle g^{2}\right\rangle=\frac{1}{16} \eta^{2}\left(1-\eta^{2}+\frac{2}{5} \eta^{3}\right) k^{2} L^{4} .
\end{gathered}
$$

One can do the integrals in the first two terms of $\rho(z)$, Eqs. (30). For the integrations, we divide the cell into five zones:

$$
\begin{array}{ll}
\text { Zone 0: } & 0 \leq z \leq \eta L / 2 \\
\text { Zone 1: } & \eta L / 2<z \leq L-\eta L / 2 \\
\text { Zone 2: } & L-\eta L / 2<z \leq L+\eta L / 2 \\
\text { Zone 3: } & L+\eta L / 2<z \leq 2 L-\eta L / 2 \\
\text { Zone 4: } & 2 L-\eta L / 2<z \leq 2 L .
\end{array}
$$

For even-numbered zones, the first integral is

$$
-\iint h=\mathbf{P}\left(\frac{n}{2}\right) \frac{1}{2}\left[\eta(2-\eta)\left(\frac{L}{2}\right)^{2}-\left(z-n \frac{L}{2}\right)^{2}\right]
$$

with $n$ the zone number. $\mathbf{P}$ is defined as follows: for any integer $m$,

$$
\mathbf{P}(m) \equiv \begin{cases}+1 & \text { if } m \text { is even; } \\ -1 & \text { if } m \text { is odd }\end{cases}
$$

For odd-numbered zones the integral is

$$
-\iint h=\mathbf{P}\left(\frac{n+1}{2}\right) \frac{1}{2} L \eta\left(z-n \frac{L}{2}\right) .
$$

As required by the definition of $\iint$, the average has been subtracted. The maximum value of $\rho_{(0)}$ (where $z=0$, implying $n=0$ ) is

$$
\rho_{(0)}^{\max }=-\left.k \iint h\right|_{0}=\frac{1}{8} \eta(2-\eta) k L^{2} .
$$

The next term in Eqs. (30) includes the integral $\iint g$. For even-numbered zones 


$$
\begin{aligned}
\iint g= & \mathbf{P}\left(\frac{n}{2}\right)\left[\eta\left(1-\frac{\eta^{2}}{2}+\frac{\eta^{3}}{8}\right)\left(\frac{L}{2}\right)^{4}\right. \\
& \left.-\frac{3}{4} \eta(2-\eta)\left(\frac{L}{2}\right)^{2}\left(z-n \frac{L}{2}\right)^{2}+\frac{1}{8}\left(z-n \frac{L}{2}\right)^{4}\right]
\end{aligned}
$$

and for odd-numbered zones

$$
\begin{aligned}
\iint g= & \mathbf{P}\left(\frac{n+1}{2}\right)\left[\left(\frac{3}{2}-\frac{\eta^{2}}{2}\right)\left(\frac{L}{2}\right)^{3}\left(z-n \frac{L}{2}\right)\right. \\
& \left.-\frac{L}{4}\left(z-n \frac{L}{2}\right)^{3}\right] .
\end{aligned}
$$

The maximum value of $\iint g$ is

$$
\left(\frac{L}{2}\right)^{4} \eta\left(1-\frac{\eta^{2}}{2}+\frac{\eta^{3}}{8}\right)
$$

These results, for any value of $\eta$, may be compared with those from Appendices $\mathrm{G}$ and $\mathrm{H}$ to determine the number of Fourier terms needed for a given accuracy in each case.

\section{APPENDIX J: NONDIMENSIONAL FORMALISM}

As mentioned in Sec. V, we have chosen in this paper to use physical units for quantities such as the axial distance $z$. The result is that the expansion parameters $k^{2}, \alpha$, and $q$ are not necessarily small numbers. To show that they are nevertheless appropriate parameters, we replace $z$ by

$$
\theta \equiv \frac{\pi z}{L}
$$

The KV equations become

$$
\begin{aligned}
& \frac{\pi^{2}}{L^{2}} a(\theta)^{\prime \prime}=-K(\theta) a+\frac{\in^{2}}{a^{3}}+\frac{2 Q}{a+b} \\
& \frac{\pi^{2}}{L^{2}} b(\theta)^{\prime \prime}=+K(\theta) b+\frac{\epsilon^{2}}{b^{3}}+\frac{2 Q}{a+b},
\end{aligned}
$$

where the primes now indicate differentiation with respect to $\theta$.

In Table I, averages are now defined by

$$
\langle f\rangle \equiv \frac{1}{2 \pi} \int_{0}^{2 \pi} f(\theta) d \theta .
$$

Similarly, the symbols $\int$ and $\iint$ refer to integration with respect to $\theta$, not $z$. Other replacements in Table I are

$$
\begin{gathered}
h(\theta) \equiv \frac{K(\theta)}{K(0)}, \\
k \equiv \frac{L^{2}}{\pi^{2}} K(0), \\
\alpha \equiv 3 \frac{L^{2}}{\pi^{2}} \frac{\in^{2}}{A^{4}}, \\
q \equiv \frac{L^{2}}{\pi^{2}} \frac{Q}{A^{2}},
\end{gathered}
$$

$$
\begin{gathered}
K^{\mathrm{eff}} \equiv \frac{L^{2}}{\pi^{2}}[K(0)]^{2}\left\langle\left[\int h\right]^{2}\right\rangle, \\
\rho_{m} \equiv h_{1} k .
\end{gathered}
$$

All these items are now dimensionless. In Eq. (J7), the quantity $\beta$ is supplanted by $\alpha$. Equations (39) and (C7) no longer apply; $\beta$ must be replaced by $\alpha$ in Eqs. (33), (40), (41), (44), and (C6) as well as four places in Appendix D and six places in Appendix E. The equations throughout this paper are otherwise unchanged, and one can verify that the expansion parameters $k^{2}, \alpha$, and $q$ are now dimensionless small numbers.

\section{APPENDIX K: ALTERNATIVE MATCHING AND INITIAL CONDITIONS}

Another method of beam matching is convenient for some simulation studies. The beam is launched at the midpoint of a quadrupole gap. Using the same lattice models as before, the matching conditions would become

$a(5 L / 2)=a(L / 2), \quad b(5 L / 2)=b(L / 2)$,

$a^{\prime}(5 L / 2)=a^{\prime}(L / 2), \quad b^{\prime}(5 L / 2)=b(L / 2)$

with initial conditions

$$
a(L / 2)=b(L / 2), \quad b^{\prime}(L / 2)=-a^{\prime}(L / 2) .
$$

This choice would be essential for the case of unequal drift spaces (only a single symmetry) but we do not consider this case here.

[1] I. M. Kapchinskij and V. V. Vladimirskij, Proceedings of the International Conference on High Energy Accelerators and Instrumentation (CERN Scientific Information Service, Geneva, 1959), p. 274.

[2] M. Reiser, Part. Accel. 8, 167 (1978).

[3] J. Struckmeier and M. Reiser, Part. Accel. 14, 227 (1984).

[4] R. C. Davidson, Physics of Nonneutral Plasmas (AddisonWesley, New York, 1990); R. C. Davidson and Q. Qian, Phys. Plasmas 1, 3104 (1994).

[5] E. P. Lee, T. J. Fessenden, and L. J. Laslett, IEEE Trans. Nucl. Sci. NS-32, 2489 (1985).

[6] E. P. Lee, Part. Accel. 52, (1996).

[7] O.A. Anderson, Lawrence Berkeley Laboratory Report No. LBL-261233 (revised), 1995.

[8] E. P. Lee, Phys. Plasmas 9, 4301 (2002).

[9] O. A. Anderson, Part. Accel. 52, 133 (1996).

[10] O. A. Anderson, PAC 2005 Proceedings, 2005, p. 3535.

[11] S. M. Lund, S. H. Chilton, and E. P. Lee, Phys. Rev. ST Accel. Beams 9, 064201 (2006).

[12] O. A. Anderson et al., Nucl. Instrum. Methods Phys. Res., Sect. B 40/41, 877 (1989).

[13] E. D. Courant and H. S. Snyder, Ann. Phys. (Leipzig) 3, 1 (1958). 